\title{
Fixed Point Theorem on Contractive Mapping in Standard 2-Normed Spaces
}

\section{Teorema Titik Tetap pada Pemetaan Kontraktif di Ruang Bernorma-2 Standar}

\author{
Salsabila Ammari ${ }^{*}$, Muh. Nur ${ }^{2 *}$, Naimah Aris ${ }^{3 *}$
}

\begin{abstract}
This paper discussed about the proof of the fixed point theorem on the standard 2-normed spaces by using completeness. The completeness of the standard 2-normed spaces is shown by defining a new norm. Two linear independent vectors on standard 2-normed spaces are used to define the new norm, namely $\|\boldsymbol{x}\|^{*}=$ $\left\|\boldsymbol{x}, \boldsymbol{a}_{\mathbf{1}}\right\|+\left\|\boldsymbol{x}, \boldsymbol{a}_{\mathbf{2}}\right\|$ which has been shown to be equivalent to standard norm.
\end{abstract}

Keywords: standard 2-normed spaces, completeness, fixed point theorem.

\begin{abstract}
Abstrak
Pada tulisan ini dibahas pembuktian teorema titik tetap pada ruang bernorma-2 standar dengan menggunakan kelengkapan. Kelengkapan dari ruang bernorma-2 standar ditunjukkan dengan mendefinisikan norma baru. Dua buah vektor bebas linier pada ruang bernorma-2 standar digunakan untuk mendefinisikan norma baru, yakni $\|\boldsymbol{x}\|^{*}=\left\|\boldsymbol{x}, \boldsymbol{a}_{\mathbf{1}}\right\|+\left\|\boldsymbol{x}, \boldsymbol{a}_{\mathbf{2}}\right\|$ yang telah ditunjukkan ekuivalen dengan norma standar.
\end{abstract}

Kata kunci: ruang bernorma-2 standar, kelengkapan, teorema titik tetap.

\section{PENDAHULUAN}

Ruang bernorma merupakan ruang vektor yang didalamnya terdapat fungsi norma dan memenuhi sifat-sifat ruang bernorma. Secara geometri, norma dapat dipandang sebagai alat ukur panjang dari suatu vektor.

Definisi 1.1 [10] Misalkan $X$ merupakan ruang vektor. Norma pada $X$ adalah fungsi $\|x\|: X \rightarrow \mathbb{R}$ sehingga untuk semua $\boldsymbol{x}, \boldsymbol{y} \in X$ dan $\alpha \in \mathbb{R}$ berlaku:

1. $\|x\| \geq 0$

2. $\|\boldsymbol{x}\|=0$ jika dan hanya jika $\boldsymbol{x}=0$

3. $\|\alpha \boldsymbol{x}\|=|\alpha|\|\boldsymbol{x}\|$

4. $\|\boldsymbol{x}+\boldsymbol{y}\| \leq\|x\|+\|\boldsymbol{y}\|$.

*Program Studi Matematika, FMIPA-UNHAS

Email Address: ${ }^{1}$ salsabilacaca.sa@gmail.com, ${ }^{2}$ muhammadnur@unhas.ac.id, ${ }^{3}$ newima@gmail.com

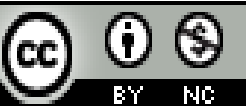

This work is licensed under a Creative Commons Attribution-NonCommercial 4.0 International License 


\section{Jurnal Matematika, Statistika \& Komputasi}

\section{Salsabila Ammari, Muh. Nur, Naimah Aris}

Ruang bernorma tidak terbatas pada ruang bernorma, terdapat pula ruang bernorma-2 hingga ruang bernorma-n untuk $n \geq 2$. Konsep tentang ruang bernorma-2 pertama kali diperkenalkan oleh Gahler pada pertengahan tahun 1960-an [7].

Definisi 1.2 [3] Misalkan $X$ adalah ruang vektor riil berdimensi $d$, dengan $d \geq 2$. Suatu fungsi bernilai riil tak negatif yang didefinisikan sebagai suatu pemetaan $\|x\|: X \times X \rightarrow \mathbb{R}$, sehingga untuk setiap $\boldsymbol{x}, \boldsymbol{y}, \boldsymbol{z} \in X$ memenuhi sifat-sifat dibawah ini:

1. $\|\boldsymbol{x}, \boldsymbol{y}\|=0$ jika dan hanya jika $x$ dan $y$ bergantung linier

2. $\|x, y\|=\|y, x\|$

3. $\|\boldsymbol{x}, \alpha \boldsymbol{y}\|=|\alpha|\|\boldsymbol{x}, \boldsymbol{y}\|$

4. $\|x, y+z\| \leq\|x, y\|+\|x, z\|$,

disebut sebagai norma-2 di $X$, dan pasangan $(X,\|.,\|$.$) disebut suatu ruang bernorma-2.$

Salah satu topik yang banyak dikembangkan oleh peneliti adalah teorema titik tetap pada pemetaan kontaktif di ruang Banach yang dilengkapi dengan norma-2. Beberapa penelitian yang telah membahas tentang topik tersebut diantaranya Gunawan [5], Nur, [14], serta Rumlawang [17]. Oleh karena itu, pada penelitian kali ini akan membahas kembali mengenai teorema titik tetap pada pemetaan kontraktif di ruang bernorma-2 standar.

\section{HASIL UTAMA}

\subsection{Ruang Bernorma-2 Standar}

Definisi 2.1 [14] Misalkan X adalah ruang hasil kali dalam dengan dimensi $d \geq 2$. Ruang vektor $X$ yang dilengkapi dengan norma-2 standar, yang didefinisikan sebagai

$$
\|x, y\|=\left|\begin{array}{ll}
\langle x, x\rangle & \langle x, y\rangle \\
\langle\boldsymbol{y}, \boldsymbol{x}\rangle & \langle\boldsymbol{y}, \boldsymbol{y}\rangle
\end{array}\right|^{\frac{1}{2}}
$$

dengan $\langle.,$.$\rangle menyatakan hasil kali dalam pada X$, merupakan ruang bernorma-2 standar.

Proposisi 2.1 Ruang bernorma-2 standar merupakan ruang bernorma-2.

Bukti.

Ambil sebarang $\boldsymbol{x}, \boldsymbol{y}, \boldsymbol{z} \in X$ dan $\alpha \in \mathbb{R}$. Akan ditunjukkan bahwa $\|.,$.$\| yang didefinisikan$ pada Definisi 2.1 memenuhi sifat-sifat norma-2.

1. Akan ditunjukkan bahwa $\|\boldsymbol{x}, \boldsymbol{y}\|=0$ jika dan hanya jika $\boldsymbol{x}$ dan $\boldsymbol{y}$ bergantung linier.

$(\Longrightarrow)$ Misalkan $\|\boldsymbol{x}, \boldsymbol{y}\|=0$. Andaikan $\boldsymbol{x}, \boldsymbol{y}$ bebas linier, dengan menggunakan metode Gram-Schmidt diperoleh $\boldsymbol{x}^{\prime}, \boldsymbol{y}^{\prime}$ yang saling ortogonal. Dengan demikian,

$$
\left|\begin{array}{ll}
\langle x, x\rangle & \langle x, y\rangle \\
\langle y, x\rangle & \langle y, y\rangle
\end{array}\right|=\left|\begin{array}{ll}
\left\langle x^{\prime}, x^{\prime}\right\rangle & \left\langle x^{\prime}, y^{\prime}\right\rangle \\
\left\langle y^{\prime}, x^{\prime}\right\rangle & \left\langle y^{\prime}, y^{\prime}\right\rangle
\end{array}\right|
$$

Karena $\boldsymbol{x}^{\prime}, \boldsymbol{y}^{\prime}$ saling ortogonal, maka $\left\langle\boldsymbol{x}^{\prime}, \boldsymbol{y}^{\prime}\right\rangle=\left\langle\boldsymbol{y}^{\prime}, \boldsymbol{x}^{\prime}\right\rangle=0$. Sehingga diperoleh,

Dengan demikian,

$$
\left|\begin{array}{ll}
\left\langle x^{\prime}, x^{\prime}\right\rangle & \left\langle x^{\prime}, y^{\prime}\right\rangle \\
\left\langle y^{\prime}, x^{\prime}\right\rangle & \left\langle y^{\prime}, y^{\prime}\right\rangle
\end{array}\right|=\left\langle x^{\prime}, x^{\prime}\right\rangle\left\langle y^{\prime}, y^{\prime}\right\rangle .
$$

$$
\left|\begin{array}{ll}
\langle x, x\rangle & \langle x, y\rangle \\
\langle y, x\rangle & \langle y, y\rangle
\end{array}\right| \neq 0 \Rightarrow\|x, y\|=\left|\begin{array}{ll}
\langle x, x\rangle & \langle x, y\rangle \\
\langle y, x\rangle & \langle y, y\rangle
\end{array}\right|^{\frac{1}{2}} \neq 0 .
$$

Hal ini bertentangan dengan pemisalan di awal, yakni $\|\boldsymbol{x}, \boldsymbol{y}\|=0$ sehingga pengandaian salah. Dengan demikian, $\boldsymbol{x}, \boldsymbol{y}$ bergantung linier. 


\section{Jurnal Matematika, Statistika \& Komputasi}

\section{Salsabila Ammari, Muh. Nur, Naimah Aris}

$(\Leftarrow)$ Misalkan $\boldsymbol{x}, \boldsymbol{y}$ bergantung linier. Artinya, dapat ditulis $\boldsymbol{x}=k \boldsymbol{y}$ dengan $k \in \mathbb{R}$. Dengan menggunakan sifat-sifat hasil kali dalam, diperoleh

$$
\|\boldsymbol{x}, \boldsymbol{y}\|^{2}=\left|\begin{array}{cc}
k^{2}\langle\boldsymbol{y}, \boldsymbol{y}\rangle & k\langle\boldsymbol{y}, \boldsymbol{y}\rangle \\
k\langle\boldsymbol{y}, \boldsymbol{y}\rangle & \langle\boldsymbol{y}, \boldsymbol{y}\rangle
\end{array}\right|
$$

Selanjutnya menggunakan sifat determinan, diperoleh

$$
\|\boldsymbol{x}, \boldsymbol{y}\|^{2}=k^{2}\left(\langle\boldsymbol{y}, \boldsymbol{y}\rangle^{2}-\langle\boldsymbol{y}, \boldsymbol{y}\rangle^{2}\right)=0 .
$$

\|. , . $\|$ memenuhi aksioma 1 norma-2.

2. Akan ditunjukkan bahwa $\|\boldsymbol{x}, \boldsymbol{y}\|=\|\boldsymbol{y}, \boldsymbol{x}\|$.

$$
\|x, y\|=\left|\begin{array}{ll}
\langle x, x\rangle & \langle x, y\rangle \\
\langle y, x\rangle & \langle y, y\rangle
\end{array}\right|^{\frac{1}{2}}=\left|\begin{array}{cc}
\langle x, x\rangle & \langle y, x\rangle \\
\langle x, y\rangle & \langle y, y\rangle
\end{array}\right|^{\frac{1}{2}}=\|y, x\| .
$$

\|. . . . memenuhi aksioma 2 norma-2.

3. Akan ditunjukkan bahwa $\|\boldsymbol{x}, \alpha \boldsymbol{y}\|=|\alpha|\|\boldsymbol{x}, \boldsymbol{y}\|$.

$$
\begin{aligned}
\|\boldsymbol{x}, \alpha \boldsymbol{y}\| & =\left|\begin{array}{cc}
\langle\boldsymbol{x}, \boldsymbol{x}\rangle & \langle\boldsymbol{x}, \alpha \boldsymbol{y}\rangle \\
\langle\alpha \boldsymbol{y}, \boldsymbol{x}\rangle & \langle\alpha \boldsymbol{y}, \alpha \boldsymbol{y}\rangle
\end{array}\right|^{\frac{1}{2}} \\
& =\left|\begin{array}{cc}
\langle\boldsymbol{x}, \boldsymbol{x}\rangle & \alpha\langle\boldsymbol{x}, \boldsymbol{y}\rangle \\
\alpha\langle\boldsymbol{y}, \boldsymbol{x}\rangle & \alpha^{2}\langle\boldsymbol{y}, \boldsymbol{y}\rangle
\end{array}\right|^{\frac{1}{2}} \\
& =\left.|\alpha| \begin{array}{cc}
\langle\boldsymbol{x}, \boldsymbol{x}\rangle & \langle\boldsymbol{x}, \boldsymbol{y}\rangle \\
\langle\boldsymbol{y}, \boldsymbol{x}\rangle & \langle\boldsymbol{y}, \boldsymbol{y}\rangle
\end{array}\right|^{\frac{1}{2}} \\
& =|\alpha|\|\boldsymbol{x}, \boldsymbol{y}\| .
\end{aligned}
$$

\|. . . . memenuhi aksioma 3 norma-2.

4. Akan ditunjukkan bahwa $\|\boldsymbol{x}+\boldsymbol{y}, \boldsymbol{z}\| \leq\|\boldsymbol{x}, \boldsymbol{z}\|+\|\boldsymbol{y}, \boldsymbol{z}\|$.

Karena

$$
\|x, y\|=\left|\begin{array}{ll}
\langle x, x\rangle & \langle x, y\rangle \\
\langle y, x\rangle & \langle y, y\rangle
\end{array}\right|^{\frac{1}{2}}=\langle x, x \mid y\rangle^{\frac{1}{2}}
$$

diperoleh,

$$
\begin{aligned}
\|x+y, z\|^{2} & =\langle\boldsymbol{x}+\boldsymbol{y}, \boldsymbol{x}+\boldsymbol{y} \mid \boldsymbol{z}\rangle . \\
& =\langle\boldsymbol{x}, \boldsymbol{x} \mid \boldsymbol{z}\rangle+2\langle\boldsymbol{x}, \boldsymbol{y} \mid \boldsymbol{z}\rangle+\langle\boldsymbol{y}, \boldsymbol{y} \mid \mathbf{z}\rangle .
\end{aligned}
$$

Dengan menggunakan Ketaksamaan Cauchy-Schwarz, maka

$$
\begin{aligned}
\|x+y, z\|^{2} & \leq\|x, z\|^{2}+2\|x, z\|\|y, z\|+\|y, z\|^{2} \\
& =(\|x, z\|+\|y, z\|) .
\end{aligned}
$$

Jadi, aksioma 4 norma-2 terpenuhi.

Dengan demikian, $\| .$, . $\|$ merupakan norma-2.

Misalkan $(X,\|.,\|$.$) ruang bernorma-2 standar dan \left\{\boldsymbol{a}_{\mathbf{1}}, \boldsymbol{a}_{\mathbf{2}}\right\}$ adalah himpunan bebas linier di $X$, didefinisikan sebuah fungsi di $X$ :

$$
\|x\|^{*}=\left\|x, a_{1}\right\|+\left\|x, a_{2}\right\| .
$$

Proposisi $2.2\left(X,\|.\|^{*}\right)$ merupakan ruang bernorma, dengan $\|.\|^{*}$ yang telah didefinisikan pada Fungsi (4.1). 


\section{Jurnal Matematika, Statistika \& Komputasi}

\section{Salsabila Ammari, Muh. Nur, Naimah Aris}

Bukti.

Ambil sebarang $\boldsymbol{x}, \boldsymbol{y} \in X$ dan $\alpha \in \mathbb{R}$. Akan ditunjukkan bahwa $\|.\|^{*}$ memenuhi sifat-sifat norma.

1. $\|x\|^{*} \geq 0$ dan $\|x\|^{*}=0$ jika dan hanya jika $\boldsymbol{x}=0$.

Karena $\|.,$.$\| merupakan norma-2, maka \left\|\boldsymbol{x}, \boldsymbol{a}_{\mathbf{1}}\right\| \geq 0$ dan $\left\|\boldsymbol{x}, \boldsymbol{a}_{\mathbf{2}}\right\| \geq 0$. Dengan demikian,

Selanjutnya,

$$
\|x\|^{*}=\left\|x, a_{1}\right\|+\left\|x, a_{2}\right\| \geq 0
$$

$(\Rightarrow)$ Misalkan $\boldsymbol{x}=0$. Diperoleh bahwa $\left\|\boldsymbol{x}, \boldsymbol{a}_{\mathbf{1}}\right\|=0$ dan $\left\|\boldsymbol{x}, \boldsymbol{a}_{\mathbf{2}}\right\|=0$. Dengan demikian,

$$
\|x\|^{*}=\left\|x, a_{1}\right\|+\left\|x, a_{2}\right\|=0 .
$$

$(\Leftarrow)$ Misalkan $\|x\|^{*}=0$ maka diperoleh,

$$
\|x\|^{*}=\left\|x, a_{1}\right\|+\left\|x, a_{2}\right\|=0
$$

Karena $\left\|\boldsymbol{x}, \boldsymbol{a}_{\mathbf{1}}\right\| \geq 0$ dan $\left\|\boldsymbol{x}, \boldsymbol{a}_{\mathbf{2}}\right\| \geq 0$, maka $\left\|\boldsymbol{x}, \boldsymbol{a}_{\mathbf{1}}\right\|=\left\|\boldsymbol{x}, \boldsymbol{a}_{\mathbf{2}}\right\|=0$. Oleh karena itu, $\boldsymbol{x}=0$.

Dengan demikian, $\|x\|^{*}=0$ jika dan hanya jika $\boldsymbol{x}=0$.

$\|.\|^{*}$ memenuhi sifat-1 norma.

2. $\|\alpha x\|^{*}=|\alpha|\|x\|^{*}$.

diperoleh,

$$
\|\alpha \boldsymbol{x}\|^{*}=\left\|\alpha \boldsymbol{x}, \boldsymbol{a}_{\mathbf{1}}\right\|+\left\|\alpha \boldsymbol{x}, \boldsymbol{a}_{\mathbf{2}}\right\|
$$

$$
\begin{aligned}
\|\alpha \boldsymbol{x}\|^{*} & =\left\|\alpha \boldsymbol{x}, \boldsymbol{a}_{\mathbf{1}}\right\|+\left\|\alpha \boldsymbol{x}, \boldsymbol{a}_{\mathbf{2}}\right\| \\
& =|\alpha|\left\|\boldsymbol{x}, \boldsymbol{a}_{\mathbf{1}}\right\|+|\alpha|\left\|\boldsymbol{x}, \boldsymbol{a}_{\mathbf{2}}\right\| \\
& =|\alpha|\left(\left\|\boldsymbol{x}, \boldsymbol{a}_{1}\right\|+\left\|\boldsymbol{x}, \boldsymbol{a}_{\mathbf{2}}\right\|\right) \\
& =|\alpha|\|\boldsymbol{x}\|^{*} .
\end{aligned}
$$

$\|.\|^{*}$ memenuhi sifat-2 norma.

3. $\|x+y\|^{*} \leq\|x\|^{*}+\|y\|^{*}$.

Karena

$$
\|x+y\|^{*}=\left\|x+y, a_{1}\right\|+\left\|x+y, a_{2}\right\|
$$

diperoleh,

$$
\begin{aligned}
\left\|x+y, a_{1}\right\|+\left\|x+y, a_{2}\right\| & \leq\left\|x, a_{1}\right\|+\left\|y, a_{1}\right\|+\left\|x, a_{2}\right\|+\left\|y, a_{2}\right\| \\
& =\left\|x, a_{1}\right\|+\left\|x, a_{2}\right\|+\left\|y, a_{1}\right\|+\left\|y, a_{2}\right\| \\
& =\|x\|^{*}+\|y\|^{*}
\end{aligned}
$$

Dengan demikian, $\|\boldsymbol{x}+\boldsymbol{y}\|^{*} \leq\|\boldsymbol{x}\|^{*}+\|\boldsymbol{y}\|^{*}$.

$\|\cdot\|^{*}$ memenuhi sifat-3 norma.

Karena $\|.\|^{*}$ memenuhi seluruh sifat norma, maka $\left(X,\|.\|^{*}\right)$ merupakan ruang bernorma.

Akibat 2.1 $\|\boldsymbol{x}\|_{1}^{*}=\left\|\boldsymbol{x}, \boldsymbol{b}_{\mathbf{1}}\right\|+\left\|\boldsymbol{x}, \boldsymbol{b}_{\mathbf{2}}\right\|$, dengan $\left\{\boldsymbol{b}_{\mathbf{1}}, \boldsymbol{b}_{\mathbf{2}}\right\}$ adalah hasil Proses Gram-Schmidt dari $\left\{\boldsymbol{a}_{1}, \boldsymbol{a}_{2}\right\}$ juga merupakan norma di $X$.

\subsection{Kekonvergenan Norma-2 Standar Menggunakan Dua Vektor Bebas Linier}

Selanjutnya akan dibuktikan bahwa norma-2 standar dapat diwakili dengan dua buah vektor bebas linier. 


\section{Jurnal Matematika, Statistika \& Komputasi}

\section{Salsabila Ammari, Muh. Nur, Naimah Aris}

Definisi 2.2 [5] Barisan $\left(x_{n}\right)$ di ruang bernorma-2 $(X,\|.,\|$.$) dikatakan konvergen ke \boldsymbol{x}$ dalam norma-2 jika dan hanya jika untuk setiap $\mathbf{z} \in X$ berlaku

$$
\lim _{n \rightarrow \infty}\left\|x_{n}-\boldsymbol{x}, \boldsymbol{z}\right\|=0
$$

Teorema 2.1 Suatu barisan $\left(x_{n}\right)$ di X konvergen ke $x$ pada norma-2 standar di X jika dan hanya jika $\lim _{n \rightarrow \infty}\left\|x_{n}-\boldsymbol{x}, \boldsymbol{a}_{\mathbf{1}}\right\|=0$ dan $\lim _{n \rightarrow \infty}\left\|x_{n}-\boldsymbol{x}, \boldsymbol{a}_{\mathbf{2}}\right\|=0$ dengan $\left\{\boldsymbol{a}_{\mathbf{1}}, \boldsymbol{a}_{\mathbf{2}}\right\}$ bebas linier.

\section{Bukti.}

Pertama-tama akan ditunjukkan bahwa $\left(x_{n}\right)$ konvergen ke $\boldsymbol{x}$ pada $X$ di norma-2 standar jika dan hanya jika $\lim _{n \rightarrow \infty}\left\|x_{n}-\boldsymbol{x}, \boldsymbol{b}_{\mathbf{1}}\right\|=0$ dan $\lim _{n \rightarrow \infty}\left\|x_{n}-\boldsymbol{x}, \boldsymbol{b}_{\mathbf{2}}\right\|=0$ dengan $\left\{\boldsymbol{b}_{\mathbf{1}}, \boldsymbol{b}_{\mathbf{2}}\right\}$ himpunan bebas linier pada $X$.

$\Leftrightarrow$ Misalkan $\left(x_{n}\right)$ konvergen ke $\boldsymbol{x}$ pada $X$ di norma-2 standar, maka $\lim _{n \rightarrow \infty}\left\|x_{n}-\boldsymbol{x}, \boldsymbol{z}\right\|=0$ untuk setiap $\boldsymbol{z} \in X$. Karena $\lim _{n \rightarrow \infty}\left\|x_{n}-\boldsymbol{x}, \boldsymbol{z}\right\|=0$ berlaku untuk setiap $\boldsymbol{z} \in X$, maka berlaku pula untuk $\boldsymbol{z}=\boldsymbol{b}_{\mathbf{1}}$ dan $\boldsymbol{z}=\boldsymbol{b}_{\mathbf{2}}$ dengan $\boldsymbol{b}_{\mathbf{1}}, \boldsymbol{b}_{\mathbf{2}} \in X$. Dengan demikian, $\lim _{n \rightarrow \infty} \| x_{n}-$ $\boldsymbol{x}, \boldsymbol{b}_{\mathbf{1}} \|=0$ dan $\lim _{n \rightarrow \infty}\left\|x_{n}-\boldsymbol{x}, \boldsymbol{b}_{2}\right\|=0$.

$(\Leftarrow)$ Akan ditunjukkan bahwa jika $\lim _{n \rightarrow \infty}\left\|x_{n}-\boldsymbol{x}, \boldsymbol{b}_{\mathbf{1}}\right\|=0$ dan $\lim _{n \rightarrow \infty}\left\|x_{n}-\boldsymbol{x}, \boldsymbol{b}_{\mathbf{2}}\right\|=0$ maka $\left(x_{n}\right)$ konvergen ke $\boldsymbol{x}$ di norma-2 standar. Jika $\left(x_{n}\right)$ konvergen ke $\boldsymbol{x}$ di norma-2 standar, maka diperoleh $\lim _{n \rightarrow \infty}\left\|x_{n}-\boldsymbol{x}, \boldsymbol{z}\right\|=0$ untuk setiap $\mathbf{z} \in X$.

Misalkan $\lim _{n \rightarrow \infty}\left\|x_{n}-\boldsymbol{x}, \boldsymbol{b}_{\boldsymbol{i}}\right\|=0$ untuk $i=1,2$. Dengan menggunakan Ketaksamaan Hadamard $\|\boldsymbol{x}, \boldsymbol{y}\| \leq\|\boldsymbol{x}\|\|\boldsymbol{y}\|$, maka diperoleh,

$$
\begin{aligned}
\left\|x_{n}-\boldsymbol{x}, \boldsymbol{z}\right\| & \leq\left\|x_{n}-\boldsymbol{x}\right\|\|\boldsymbol{z}\| . \\
& \leq\left[\left\|x_{n}-\boldsymbol{x}, \boldsymbol{b}_{1}\right\|+\left\|x_{n}-\boldsymbol{x}, \boldsymbol{b}_{2}\right\|\right]\|\boldsymbol{z}\| .
\end{aligned}
$$

Karena $\left\|x_{n}-\boldsymbol{x}, \boldsymbol{b}_{\boldsymbol{i}}\right\| \rightarrow 0$ untuk $n \rightarrow \infty$, maka $\lim _{n \rightarrow \infty}\left\|x_{n}-\boldsymbol{x}, \boldsymbol{z}\right\|=0$. Dengan demikian, $\left(x_{n}\right)$ konvergen ke $\boldsymbol{x}$ di norma-2 standar.

Selanjutnya, ambil $k, l_{1}, l_{2} \in \mathbb{R}$, akan ditunjukkan bahwa $\lim _{n \rightarrow \infty}\left\|x_{n}-\boldsymbol{x}, \boldsymbol{a}_{\mathbf{1}}\right\|=0$ dan $\lim _{n \rightarrow \infty}\left\|x_{n}-\boldsymbol{x}, \boldsymbol{a}_{\mathbf{2}}\right\|=0$ jika dan hanya jika $\lim _{n \rightarrow \infty}\left\|x_{n}-\boldsymbol{x}, \boldsymbol{b}_{\mathbf{1}}\right\|=0$ dan $\lim _{n \rightarrow \infty} \| x_{n}-$ $\boldsymbol{x}, \boldsymbol{b}_{2} \|=0$.

Karena $\left\{\boldsymbol{b}_{\mathbf{1}}, \boldsymbol{b}_{2}\right\}$ merupakan hasil Gram-Schmidt dari $\left\{\boldsymbol{a}_{\mathbf{1}}, \boldsymbol{a}_{\mathbf{2}}\right\}$, maka dapat ditulis

$$
\boldsymbol{b}_{1}=k \boldsymbol{a}_{1} ; \boldsymbol{b}_{2}=l \boldsymbol{a}_{1}+l \boldsymbol{a}_{2} \text {. }
$$

Berdasarkan sifat norma-2 diperoleh,

$$
\left\|x, b_{1}\right\|=\left\|x, k a_{1}\right\|=|k|\left\|x, a_{1}\right\|
$$

dan

$$
\left\|\boldsymbol{x}, \boldsymbol{b}_{2}\right\| \leq\left|l_{1}\right|\left\|\boldsymbol{x}, \boldsymbol{a}_{1}\right\|+\left|l_{2}\right||| \boldsymbol{x}, \boldsymbol{a}_{2} \| .
$$

Misalkan $s_{1}=\frac{1}{l_{2}} ; s_{2}=\frac{l_{1}}{l_{2} k}$, maka diperoleh,

dan

$$
\left\|x, a_{1}\right\|=\left\|x, \frac{b_{1}}{k}\right\|=\left|\frac{1}{k}\right|\left\|x, b_{1}\right\|
$$

$$
\begin{aligned}
\left\|\boldsymbol{x}, \boldsymbol{a}_{2}\right\| & =\left\|\boldsymbol{x}, s_{1} \boldsymbol{b}_{2}-s_{2} \boldsymbol{b}_{1}\right\|=\left\|\boldsymbol{x}, s_{1} \boldsymbol{b}_{2}+\left(-s_{2} \boldsymbol{b}_{1}\right)\right\| \\
& \leq\left\|\boldsymbol{x}, s_{1} \boldsymbol{b}_{2}\right\|+\left\|\boldsymbol{x},-s_{2} \boldsymbol{b}_{1}\right\|=\left|s_{1}\right|\left\|\boldsymbol{x}, \boldsymbol{b}_{2}\right\|+\left|s_{2}\right|\left\|\boldsymbol{x}, \boldsymbol{b}_{1}\right\| .
\end{aligned}
$$

$(\Rightarrow)$ Misalkan $\lim _{n \rightarrow \infty}\left\|x_{n}-\boldsymbol{x}, \boldsymbol{a}_{\mathbf{1}}\right\|=0$ dan $\lim _{n \rightarrow \infty}\left\|x_{n}-\boldsymbol{x}, \boldsymbol{a}_{\mathbf{2}}\right\|=0$. Perhatikan bahwa,

$$
\lim _{n \rightarrow \infty}\left\|x_{n}-\boldsymbol{x}, \boldsymbol{b}_{\mathbf{1}}\right\|=\lim _{n \rightarrow \infty}|k|\left\|x_{n}-\boldsymbol{x}, \boldsymbol{a}_{\mathbf{1}}\right\|=|k| \lim _{n \rightarrow \infty}\left\|x_{n}-\boldsymbol{x}, \boldsymbol{a}_{\mathbf{1}}\right\|=0,
$$

sehingga diperoleh $\lim _{n \rightarrow \infty}\left\|x_{n}-\boldsymbol{x}, \boldsymbol{b}_{\mathbf{1}}\right\|=0$. Selanjutnya perhatikan,

$$
\begin{aligned}
\lim _{n \rightarrow \infty}\left\|x_{n}-\boldsymbol{x}, \boldsymbol{b}_{2}\right\| & \leq \lim _{n \rightarrow \infty}\left(\left|l_{1}\right|\left\|x_{n}-\boldsymbol{x}, \boldsymbol{a}_{1}\right\|\right)+\lim _{n \rightarrow \infty}\left(\left|l_{2}\right|\left\|x_{n}-\boldsymbol{x}, \boldsymbol{a}_{2}\right\|\right) \\
& =\left|l_{1}\right| \lim _{n \rightarrow \infty}\left\|x_{n}-\boldsymbol{x}, \boldsymbol{a}_{\mathbf{1}}\right\|+\left|l_{2}\right| \lim _{n \rightarrow \infty}\left\|x_{n}-\boldsymbol{x}, \boldsymbol{a}_{\mathbf{2}}\right\| \\
& =0 .
\end{aligned}
$$

Karena $\left\|x_{n}-\boldsymbol{x}, \boldsymbol{b}_{\mathbf{2}}\right\| \geq 0$, maka $\lim _{n \rightarrow \infty}\left\|x_{n}-\boldsymbol{x}, \boldsymbol{b}_{\mathbf{2}}\right\|=0$.

$(\Leftarrow)$ Misalkan $\lim _{n \rightarrow \infty}\left\|x_{n}-\boldsymbol{x}, \boldsymbol{b}_{\mathbf{1}}\right\|=0$ dan $\lim _{n \rightarrow \infty}\left\|x_{n}-\boldsymbol{x}, \boldsymbol{b}_{\mathbf{2}}\right\|=0$. Dengan cara yang sama, diperoleh $\lim _{n \rightarrow \infty}\left\|x_{n}-\boldsymbol{x}, \boldsymbol{a}_{\mathbf{1}}\right\|=0$ dan $\lim _{n \rightarrow \infty}\left\|x_{n}-\boldsymbol{x}, \boldsymbol{a}_{\mathbf{2}}\right\|=0$. 


\section{Jurnal Matematika, Statistika \& Komputasi}

\section{Salsabila Ammari, Muh. Nur, Naimah Aris}

Dengan demikian, barisan $\left(x_{n}\right)$ di $X$ konvergen ke $\boldsymbol{x}$ pada norma-2 standar di $X$ jika dan hanya jika $\lim _{n \rightarrow \infty}\left\|x_{n}-\boldsymbol{x}, \boldsymbol{a}_{\mathbf{1}}\right\|=0$ dan $\lim _{n \rightarrow \infty}\left\|x_{n}-\boldsymbol{x}, \boldsymbol{a}_{\mathbf{2}}\right\|=0$.

Akibat 2.2 Ruang X yang dilengkapi $\|.\|^{*}$ lengkap jika dan hanya jika X yang dilengkapi $\|.\|_{1}^{*}$ lengkap.

\subsection{Kelengkapan Norma-2 Standar}

Selanjutnya, akan dibahas mengenai kelengkapan norma-2 standar dengan menggunakan norma biasa.

Proposisi $2.3\|.\|_{1}^{*}$ ekuivalen dengan norma standar $\|$.$\| di X$, yakni $\|x\|=\langle x, x\rangle^{\frac{1}{2}}$. Artinya,

\section{Bukti.}

$$
\|x\| \leq\|x\|_{1}^{*} \leq 2\|x\| \text {. }
$$

Ambil sebarang $\boldsymbol{x} \in X$. Karena $\left\{\boldsymbol{b}_{\mathbf{1}}, \boldsymbol{b}_{2}\right\}$ merupakan hasil Gram-Schmidt dari $\left\{\boldsymbol{a}_{\mathbf{1}}, \boldsymbol{a}_{\mathbf{2}}\right\}$, maka $\left\{\boldsymbol{b}_{1}, \boldsymbol{b}_{\mathbf{2}}\right\}$ merupakan himpunan ortonormal pada $X$, diperoleh $\left\|\boldsymbol{x}, \boldsymbol{b}_{\mathbf{1}}\right\|^{2}=\|\boldsymbol{x}\|^{2}-\left\langle\boldsymbol{x}, \boldsymbol{b}_{\mathbf{1}}\right\rangle^{2}$ dan $\left\|\boldsymbol{x}, \boldsymbol{b}_{2}\right\|^{2}=\|\boldsymbol{x}\|^{2}-\left\langle\boldsymbol{x}, \boldsymbol{b}_{2}\right\rangle^{2}$.

Berdasarkan Ketaksamaan Bessel $\sum_{i=1}^{2}\left\langle\boldsymbol{x}, \boldsymbol{b}_{\boldsymbol{i}}\right\rangle^{2} \leq\|\boldsymbol{x}\|^{2}$, diperoleh

Karena

$$
\begin{aligned}
\|x\|^{2} & \leq 2\|x\|^{2}-\sum_{i=1}^{2}\left\langle x, b_{i}\right\rangle^{2}=\|x\|^{2}-\left\langle x, b_{1}\right\rangle^{2}+\|x\|^{2}-\left\langle x, b_{2}\right\rangle^{2} \\
& =\left\|x, b_{1}\right\|^{2}+\left\|x, b_{2}\right\|^{2} .
\end{aligned}
$$

maka

$$
\left(\left\|x, b_{1}\right\|+\left\|x, b_{2}\right\|\right)^{2} \geq\left\|x, b_{1}\right\|^{2}+\left\|x, b_{2}\right\|^{2},
$$

$$
\begin{aligned}
\|x\|^{2} & \leq\left\|x, b_{1}\right\|^{2}+\left\|x, b_{2}\right\|^{2} \leq\left(\left\|x, b_{1}\right\|+\left\|x, b_{2}\right\|\right)^{2} \\
\|x\| & \leq\left\|x, b_{1}\right\|+\left\|x, b_{2}\right\|=\|x\|_{1}^{*} .
\end{aligned}
$$

Selanjutnya, perhatikan bahwa $\|\boldsymbol{x}\|_{1}^{*}=\left\|\boldsymbol{x}, \boldsymbol{b}_{\mathbf{1}}\right\|+\left\|\boldsymbol{x}, \boldsymbol{b}_{\mathbf{2}}\right\|=\sum_{i=1}^{2}\left\|\boldsymbol{x}, \boldsymbol{b}_{\boldsymbol{i}}\right\|$. Dengan menggunakan Ketaksamaan Hadamard $\|\boldsymbol{x}, \boldsymbol{y}\| \leq\|\boldsymbol{x}\|\|\boldsymbol{y}\|$ diperoleh,

$$
\sum_{i=1}^{2}\left\|x, b_{i}\right\| \leq\|x\|\left(\left\|b_{1}\right\|+\left\|b_{2}\right\|\right) .
$$

Ingat bahwa $\left\{\boldsymbol{b}_{1}, \boldsymbol{b}_{2}\right\}$ merupakan himpunan ortonormal pada $X$ sehingga $\left\|\boldsymbol{b}_{\boldsymbol{i}}\right\|=1$ untuk $i=1,2$. Dengan demikian,

$$
\sum_{i=1}^{2}\left\|x, b_{i}\right\| \leq\|x\|(1+1)=2\|x\| .
$$

Jadi, diperoleh bahwa $\|x\| \leq\|x\|_{1}^{*} \leq 2\|x\|$.

$\therefore\|.\|_{1}^{*}$ ekuivalen dengan $\|$.$\| .$

Definisi 2.3 [5] Barisan $\left(x_{n}\right)$ dikatakan Cauchy apabila untuk setiap $\boldsymbol{z} \in X$ dan untuk setiap $\varepsilon>0$ terdapat $n_{0} \in \mathbb{N}$ sehingga $\left\|x_{m}-x_{n}, \boldsymbol{z}\right\|<\varepsilon$ untuk setiap $m, n \geq n_{0}$. Jika setiap barisan Cauchy di $(X,\|.,\|$.$) konvergen ke suatu \boldsymbol{x} \in X$, maka X dikatakan lengkap.

Teorema 2.2 Barisan $\left(x_{n}\right)$ di $X$ konvergen pada ruang bernorma-2 standar $(X,\|.,\|$.$) jika dan$ hanya jika barisan itu konvergen di ruang bernorma $(X,\|\|$.$) , dan barisan \left(x_{n}\right)$ di $X$ Cauchy di ruang bernorma-2 standar $(X,\|.,\|$.$) jika dan hanya jika barisan itu Cauchy di ruang bernorma$ $(X,\|\cdot\|)$.

Bukti. 


\section{Jurnal Matematika, Statistika \& Komputasi}

\section{Salsabila Ammari, Muh. Nur, Naimah Aris}

Pertama-tama akan ditunjukkan bahwa barisan $\left(x_{n}\right)$ di $X$ konvergen pada ruang bernorma-2 standar $(X,\|.,\|$.$) jika dan hanya jika barisan itu konvergen di ruang bernorma$ $(X,\|\cdot\|)$.

$\Leftrightarrow$ Misalkan diketahui $\left(x_{n}\right)$ konvergen ke $\boldsymbol{x}$ dalam ruang norma-2 $(X,\|.,\|$.$) . Berdasarkan$ Teorema 2.1, diperoleh $\lim _{n \rightarrow \infty}\left\|x_{n}-\boldsymbol{x}, \boldsymbol{b}_{\mathbf{1}}\right\|=0$ dan $\lim _{n \rightarrow \infty}\left\|x_{n}-\boldsymbol{x}, \boldsymbol{b}_{\mathbf{2}}\right\|=0$. Perhatikan bahwa,

$$
\begin{aligned}
\lim _{n \rightarrow \infty}\left\|x_{n}-\boldsymbol{x}\right\|_{1}^{*} & =\lim _{n \rightarrow \infty}\left(\left\|x_{n}-\boldsymbol{x}, \boldsymbol{b}_{\mathbf{1}}\right\|+\left\|x_{n}-\boldsymbol{x}, \boldsymbol{b}_{\mathbf{2}}\right\|\right) \\
& =\lim _{n \rightarrow \infty}\left\|x_{n}-\boldsymbol{x}, \boldsymbol{b}_{\mathbf{1}}\right\|+\lim _{n \rightarrow \infty}\left\|x_{n}-\boldsymbol{x}, \boldsymbol{b}_{\mathbf{2}}\right\| \\
& =0 .
\end{aligned}
$$

Karena $\|.\|_{1}^{*}$ ekuivalen dengan $\|$.$\| , maka \lim _{n \rightarrow \infty}\left\|x_{n}-\boldsymbol{x}\right\|=0$. Berdasarkan definisi kekonvergenan, maka $\left(x_{n}\right)$ konvergen ke $\boldsymbol{x}$ pada ruang bernorma $(X,\|\|$.$) .$

$(\Leftarrow)$ Ambil sebarang $\boldsymbol{z} \in X$. Misalkan $\left(x_{n}\right)$ konvergen ke $\boldsymbol{x}$ dalam ruang norma $(X, \|$. $\|)$, maka diperoleh bahwa untuk setiap $\varepsilon>0$ terdapat $n_{0} \in \mathbb{N}$, sehingga $\left\|x_{n}-\boldsymbol{x}\right\|<\frac{\varepsilon}{\|z\|}$ untuk $n \geq n_{0}$. Menurut Ketaksamaan Hadamard, diperoleh,

$$
\left\|x_{n}-\boldsymbol{x}, \boldsymbol{z}\right\| \leq\left\|x_{n}-\boldsymbol{x}\right\|\|\mathbf{z}\|<\varepsilon
$$

untuk setiap $n \geq n_{0}$. Dengan demikian, $\left(x_{n}\right)$ konvergen ke $\boldsymbol{x}$ dalam ruang norma-2 $(X,\|.,\|$.$) .$

Selanjutnya akan ditunjukkan bahwa barisan $\left(x_{n}\right)$ di $X$ Cauchy di ruang bernorma-2 $\operatorname{standar}(X,\|.,\|$.$) jika dan hanya jika barisan itu Cauchy di ruang bernorma (X,\|\|$.$) .$

$\Leftrightarrow)$ Misalkan diketahui $\left(x_{n}\right)$ Cauchy di ruang norma-2 $(X, \| ., \ldots)$, diperoleh bahwa $\lim _{m, n \rightarrow \infty}\left\|x_{m}-x_{n}, \boldsymbol{z}\right\|=0$. Berdasarkan Teorema 2.1 maka $\lim _{m, n \rightarrow \infty}\left\|x_{m}-x_{n}, \boldsymbol{b}_{\mathbf{1}}\right\|=0$ dan $\lim _{m, n \rightarrow \infty}\left\|x_{m}-x_{n}, \boldsymbol{b}_{2}\right\|=0$. Perhatikan bahwa,

$$
\begin{aligned}
\lim _{m, n \rightarrow \infty}\left\|x_{m}-x_{n}\right\|_{1}^{*} & =\lim _{m, n \rightarrow \infty}\left(\left\|x_{m}-x_{n}, \boldsymbol{b}_{\mathbf{1}}\right\|+\left\|x_{m}-x_{n}, \boldsymbol{b}_{\mathbf{2}}\right\|\right) \\
& =\lim _{m, n \rightarrow \infty}\left\|x_{m}-x_{n}, \boldsymbol{b}_{\mathbf{1}}\right\|+\lim _{m, n \rightarrow \infty}\left\|x_{m}-x_{n}, \boldsymbol{b}_{\mathbf{2}}\right\| \\
& =0 .
\end{aligned}
$$

Karena sebelumnya telah ditunjukkan bahwa $\|.\|_{1}^{*}$ ekuivalen dengan $\|$.$\| , maka$ $\lim _{m, n \rightarrow \infty}\left\|x_{m}-x_{n}\right\|=0$. Atau dapat ditulis, terdapat $\varepsilon>0$ untuk setiap $n_{0} \in \mathbb{N}$ sehingga $\left\|x_{m}-x_{n}\right\|<\varepsilon$ untuk $m, n \geq n_{0}$. Akibatnya $\left(x_{n}\right)$ Cauchy di ruang bernorma $(X,\|\|$.$) .$

$(\Leftarrow)$ Ambil sebarang $\boldsymbol{z} \in X$. Misalkan $\left(x_{n}\right)$ Cauchy di ruang bernorma $(X,\|\|$.$) , maka \| x_{m}-$ $x_{n} \|<\frac{\varepsilon}{\|z\|}$ untuk $m, n \geq n_{0}$. Menurut Ketaksamaan Hadamard, diperoleh,

$$
\left\|x_{m}-x_{n}, \mathbf{z}\right\| \leq\left\|x_{m}-x_{n}\right\|\|z\|<\varepsilon
$$

untuk setiap $m, n \geq n_{0}$. Dengan demikian, $\left(x_{n}\right)$ juga Cauchy di ruang bernorma-2 $(X,\|.,\|$.$) .$

$\therefore$ Teorema 2.2 terbukti.

Akibat 2.3 Ruang bernorma-2 standar $(X,\|.,\|$.$) lengkap jika dan hanya jika ruang bernorma$ $\operatorname{standar}(X,\|\|$.$) adalah lengkap.$

\subsection{Teorema Titik Tetap pada Pemetaan Kontraktif di Ruang Bernorma-2 Standar}

Dengan menggunakan hasil-hasil pada subbab sebelumnya, akan dibuktikan teorema titik tetap pada pemetaan kontraktif di ruang bernorma-2 standar.

Teorema 2.3 Misalkan (X, $\|.,\|$.$) merupakan ruang Banach-2. \left\{\boldsymbol{a}_{\mathbf{1}}, \boldsymbol{a}_{\mathbf{2}}\right\}$ merupakan himpunan bebas linier dan $T: X \rightarrow X$ memenuhi $\left\|T_{\boldsymbol{x}}-T_{\boldsymbol{y}}, \boldsymbol{a}_{\boldsymbol{i}}\right\| \leq k\left\|\boldsymbol{x}-\boldsymbol{y}, \boldsymbol{a}_{\boldsymbol{i}}\right\|$ untuk setiap $\boldsymbol{x}, \boldsymbol{y}, \boldsymbol{a}_{\boldsymbol{i}} \in X$ dimana $i=1,2$ dan $0<k<1$ konstan, maka T mempunyai titik tetap yang tunggal.

\section{Bukti.}




\section{Jurnal Matematika, Statistika \& Komputasi \\ Salsabila Ammari, Muh. Nur, Naimah Aris}

Ambil sebarang himpunan bebas linier $\left\{\boldsymbol{a}_{1}, \boldsymbol{a}_{2}\right\}$ di $(X,\|.,\|$.$) , pada Proposisi 2.2$ telah ditunjukkan bahwa $\|\boldsymbol{x}\|^{*}=\left\|\boldsymbol{x}, \boldsymbol{a}_{\mathbf{1}}\right\|+\left\|\boldsymbol{x}, \boldsymbol{a}_{2}\right\|$ dan $\|\boldsymbol{x}\|_{1}^{*}=\left\|\boldsymbol{x}, \boldsymbol{b}_{\mathbf{1}}\right\|+\left\|\boldsymbol{x}, \boldsymbol{b}_{2}\right\|$ merupakan norma pada $X$, dengan $\left\{\boldsymbol{b}_{1}, \boldsymbol{b}_{2}\right\}$ adalah hasil Gram-Schmidt dari $\left\{\boldsymbol{a}_{1}, \boldsymbol{a}_{2}\right\}$. Karena $(X,\|.,\|$. merupakan ruang bernorma-2 yang lengkap, maka berdasarkan Akibat 2.3, $(X,\|\|$.$) adalah ruang$ bernorma yang lengkap. Diketahui pula dari Proposisi 2.3 bahwa $\|$.$\| ekuivalen dengan \|.\|_{*}^{1}$, akibatnya $\|.\|_{*}^{1}$ lengkap. Lebih jauh pada Akibat 2.2, $\|.\|^{*}$ adalah lengkap.

Selanjutnya, misalkan $\left\|T_{\boldsymbol{x}}-T_{\boldsymbol{y}}, \boldsymbol{a}_{\boldsymbol{i}}\right\| \leq k\left\|\boldsymbol{x}-\boldsymbol{y}, \boldsymbol{a}_{\boldsymbol{i}}\right\|$ untuk setiap $\boldsymbol{x}, \boldsymbol{y}, \boldsymbol{a}_{\boldsymbol{i}} \in X$ dimana $i=1,2$ dan $0<k<1$ konstan. Perhatikan bahwa untuk setiap $\boldsymbol{x}, \boldsymbol{y} \in X$,

$$
\begin{aligned}
\left\|T_{x}-T_{y}\right\|^{*} & =\left\|T_{x}-T_{y}, \boldsymbol{a}_{1}\right\|+\left\|T_{x}-T_{\boldsymbol{y}}, \boldsymbol{a}_{2}\right\| \\
& \leq k\left\|\boldsymbol{x}-\boldsymbol{y}, \boldsymbol{a}_{1}\right\|+k\left\|\boldsymbol{x}-\boldsymbol{y}, \boldsymbol{a}_{2}\right\| \\
& =k\|\boldsymbol{x}-\boldsymbol{y}\|^{*} .
\end{aligned}
$$

Karena $\left\|T_{x}-T_{\boldsymbol{y}}\right\|^{*} \leq k\|\boldsymbol{x}-\boldsymbol{y}\|^{*}$, maka $T$ kontraktif terhadap $\|.\|^{*}$. Karena $T$ kontraktif terhadap $\|.\|^{*}$ dan $\left(X,\|.\|^{*}\right)$ lengkap, maka $T$ memiliki titik tetap yang tunggal.

\section{KESIMPULAN}

Kekonvergenan dan kelengkapan dari ruang bernorma-2 standar ditunjukkan dengan mendefinisikan norma baru. Dua buah vektor bebas linier pada norma-2 standar digunakan untuk mendefinisikan norma baru, yakni $\|\boldsymbol{x}\|^{*}=\left\|\boldsymbol{x}, \boldsymbol{a}_{\mathbf{1}}\right\|+\left\|\boldsymbol{x}, \boldsymbol{a}_{\mathbf{2}}\right\|$ yang telah ditunjukkan ekuivalen dengan norma standar yakni norma yang diinduksi dari hasil kali dalam. Ekuivalensi dan kelengkapan yang telah ditunjukkan digunakan untuk membuktikan teorema titik tetap.

\section{DAFTAR PUSTAKA}

[1] Anton, H. Rorres, C., 2005. Elementary Linear Algebra. Newyork: John Wiley \& Sons Inc.

[2] Conway, J., 1997. A Course in Functional Analysis. Newyork: Springer-Verlag.

[3] Gähler, S., 1964. Lineare 2-Nomierte Räume. Math Nachr, Vol. 28, No. 1, 1-43.

[4] Gunawan, H., 2001. The Space of p-Summable Sequences and Its Natural n-Norm. Bull Australia Math Soc, Vol. 64, No. 1, 137-147.

[5] Gunawan, H., Mashadi. 2001. On Finite Dimensional 2-Normed Spaces. Soochow Journal of Mathematics, Vol. 27, No. 3, 321-329.

[6] Haase, M., 2010. Functional Analysis: An Elementary Introduction. Rhode Island : American Mathematical Society Providance.

[7] Handayani, A., 2013. Skripsi: Barisan pada Ruang Norm-2. Jurusan Matematika Fakultas Sains dan Teknologi UIN Malang.

[8] Harikrishnan, K., Ravindran, T., 2011. Some Properties of Accretive Operators in Linier 2Normed Space. Int. Mathematical Forum, Vol. 3, No. 59, 2941-2947.

[9] Imrona, M., 2002. Aljabar Linier Elementer. Bandung: Sekolah Tinggi Teknologi Telkom. 


\section{Jurnal Matematika, Statistika \& Komputasi}

Salsabila Ammari, Muh. Nur, Naimah Aris

[10] Kreyszig, E., 1978. Introductory Functional Analysis With Application. Newyork: John Wiley dan Sons Inc.

[11] Lidiyani, Y., Shiddiq, M., 2017. Teorema Titik Tetap untuk Pemetaan Kontraksi-F yang Diperumum pada Ruang Seperti-Metrik-b. Jurnal Matematika Murni dan Terapan “Epsilon”, Vol. 11, No.2, 36-46.

[12] Manuhutu, J., Lesnussa, A., Batkunde, H., 2014. Ruang Norm-2 dan Ruang Hasil Kali Dalam-2. Jurnal Matematika Integratif, Vol. 10, No. 2, 139-145.

[13] Nur, M., 2011. Tesis: Teorema Titik Tetap di Ruang Norm-n Standar. Institut Teknologi Bandung.

[14] Nur, M., 2012. Teorema Titik Tetap di Ruang Norm-2 Standar. Jurnal Matematika Statistika dan Komputasi, Vol. 13, No. 1, 1-6.

[15] Resmawan., 2019. Materi Kuliah Aljabar Linear. Math UNG, Universitas Negeri Gorontalo.

[16] Rudin, W., 1991. Functional Analysis. Singapore: McGraw-Hill.

[17] Rumlawang, F. Y., 2020. Fixed Point Theorem in 2-Normed Spaces. Tensor Pure and Applied Mathematic's Journal, Vol. 1, No. 1, 41-46.

[18] Rynne, B.P., Youngson, M. A., 2000. Linier Functional Analysis. London: Springer-Verlag. 